\title{
Optimal Data Collection For A 2-LeVel BALANCED WSN
}

\author{
John R Rankin \\ Charles Sturt University Study Group Melbourne, Australia
}

\begin{abstract}
Battery life is a drawback of Wireless Sensor Networks (WSNs) but careful management of the network can provide optimum performance before batteries need replacing. This paper models and analyses the productivity and energy consumption of a 2-level balanced WSN. By optimizing the energy versus time curve first with respect to quiescent periods we obtain a curve of rest length as a function of the number of level two nodes and then by secondly optimizing this curve with respect to the number of remote nodes per level one sensor node, an overall optimum network management strategy is achieved. Programs were written to display the productivity and longevity curves and determine the optimum point if it exists.
\end{abstract}

\section{KEYWORDS}

Wireless Sensor Network, Network Architecture, Optimization, Productivity, Longevity

\section{INTRODUCTION}

Wireless Sensor Networks have found widespread acceptance in remote surveillance applications $[2,3,5]$ because the nodes are now cheap, sensors are high quality and wireless transmission eliminates the inconvenience and expense of cabling. The main disadvantage of the WSN is battery life: data collection can only continue so long as the battery power level remains above a minimum value. This aspect was addressed in a study by Hong A $N$ et al [1] and the current paper models a WSN architecture taking their results into consideration. Efforts for increasing WSN efficiency were also considered by other authors $[4,6,7]$.

Here we will assume that the WSN hasone mains powered Collector Node, $\mathrm{CN}$, and $\mathrm{N}$ identical battery operated nodes arranged hierarchically as in Figure 1sending data collected at each node up the hierarchy node by node to reach the Collector Node at the apex of the hierarchy. In this work we analyse network effectiveness for 1 and 2 level hierarchical architectures.

Each of the N sensor nodes of the WSN can send its sample data in a TDMA timeslot of preset fixed length $\Delta$ up one level of the hierarchy to its designated node for relaying its data onwards. The Collector Node CN at the hierarchy apex uses timeslot 0 for transmissions and Sensor Node $\mathrm{SN}$ i uses timeslot $\mathrm{i}$ for $\mathrm{i}=1$ to $\mathrm{N}$ for its transmissions. $\mathrm{CN}$ is listening for transmissions in timeslots $\mathrm{i}=1$ to $\mathrm{N}$ and $\mathrm{SN} \mathrm{i}$ is listening in all timeslots except timeslot $\mathrm{i}$. This means that the communications cycle time of the WSN is $\mathrm{T}=(\mathrm{N}+1) \Delta$. Compared with this cycle time, sensor sampling takes a long time denoted $t_{\mathrm{S}}=\mathrm{ZT}$ where $\mathrm{Z}$ is a large positive integer and typically $\mathrm{Z} \geq$ 100. During sampling operations, each $S N$ goes through its own cycle of length $t_{\mathrm{T}}$. First it uses $Z$ network cycles to sample the environment. Then it uses the next network cycle to transmit the sampled data. After this the SN rests for another R network cycles and this allows the battery to recover. Altogether the $\mathrm{SN}$ cycle consists of $(\mathrm{Z}+1+\mathrm{R})$ network cycles in which the $\mathrm{SN}$ is active for a time $t_{A}$ and passive for a time $t_{P}$. Therefore: 


$$
\begin{aligned}
& t_{T}=t_{A}+t_{P} \\
& t_{A}=Z T+\Delta \\
& t_{P}=N \Delta+R T
\end{aligned}
$$

In the formulas used, $\Delta, \mathrm{Z}, \mathrm{N}$ and $\mathrm{T}$ are constants of the system and our main focus is on variables $\mathrm{N}_{1}$ and R.Eqn(1) shows that $t_{A}$ is constant but $t_{P}$ and hence $t_{T}$ is linearly dependent on $R$ with positive coefficients.

$$
\begin{aligned}
& \square=\text { Collector Node } \\
& \square=\text { Sensor Node }
\end{aligned}
$$

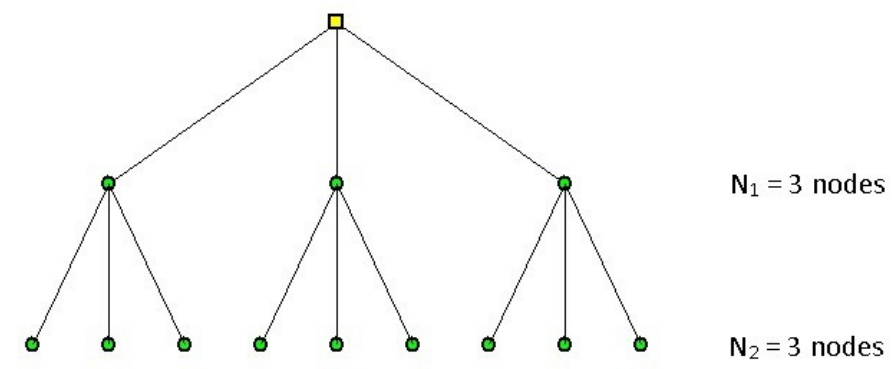

Figure 1 Hierarchical WSN architecture used in this research.

\section{1 -LeVel Energy CONSUMPTiOn MODELling}

In the case of a 1-level WSN, we have $\mathrm{N}=\mathrm{N}_{1}$ sensor nodes (SNs) surrounding the collector node $(\mathrm{CN})$ each within the maximum efficient radio reach $\mathrm{r}$ of the $\mathrm{CN}$ and each $\mathrm{SN}$ transmitting directly to the $C N$. Let $E_{S}$ be the initial battery level when sampling operations begin and $E_{0}$ the minimal operational energy. Using $\varepsilon_{\mathrm{A}}$ to denote the active energy consumption rate and $\varepsilon_{\mathrm{P}}$ to denote the passive energy consumption rate during $\mathrm{SN}$ sampling operation the energy consumption per SN per SN cycle is therefore:

$$
E_{T}=\varepsilon_{A} t_{A}+\varepsilon_{P} t_{P}
$$

From Eqn(1) and (2) it follows that $\mathrm{E}_{\mathrm{T}}$ is linearly dependent on $\mathrm{R}$ with positive coefficients. From this we can determine the WSN productivity $\mathrm{P}$ (total number of samples reported) and longevity $\mathrm{L}$ (duration of the WSN after initialization) according to these formulas:

$$
\begin{aligned}
& P=N \frac{\left(E_{S}-E_{0}\right)}{E_{T}} \\
& L=\frac{\left(E_{S}-E_{0}\right) t_{T}}{E_{T}}
\end{aligned}
$$

In Eqn(3) and (4) N, $E_{S}$ and $E_{0}$ are constants but $E_{T}$ and $t_{T}$ are linearly dependent on $R$ with positive coefficients. These formulas are plotted in Figures 2a. The blue line is the productivity plot with $\mathrm{P}$ on the vertical axis increasing upwards and $\mathrm{R}$ on the horizontal axis increasing to the right.It is hyperbolic in form decreasing from an initial maximum towards zero according to Eqn(3). The green line is the longevity plot with $L$ on the vertical axis increasing upwards and $R$ on the horizontal axis increasing to the right. According to Eqn(4) this curve is a rational linear with positive coefficients which means that it rises from zero to an asymptote and therefore 
doesn't have a turning point. Thus both curveshave no peak value for any R. In Eqn(3), the productivity falls off hyperbolically with $\mathrm{R}$ and has no peak. In Eqn (4), the longevity increases asymptotically as $\mathrm{R}$ increases again with no peak. The only parameter we can use to optimize a 1level architecture of fixed $\mathrm{N}$ is $\mathrm{R}$ but no peak is achieved for any finite value of $\mathrm{R}$. The 1-level architecture is therefore unoptimizable. The results are different for a 2-level WSN however.

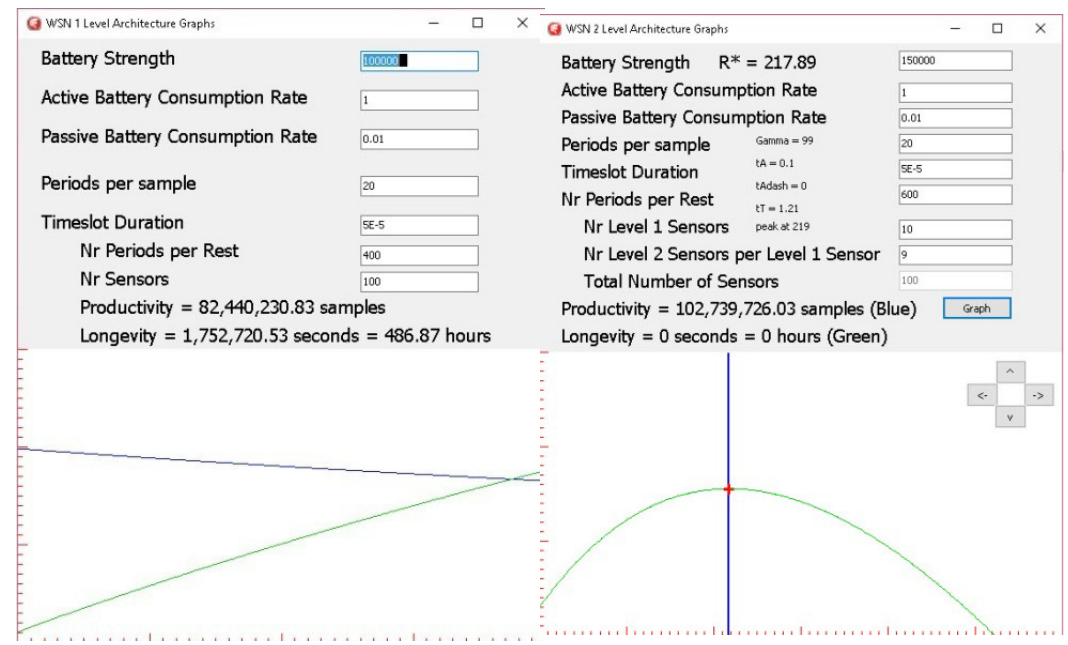

Figure 2a Program for 1 level WSN graphs. Figure 2b Program for 2 level WSN graphs.

\subsection{LEVEL ENERGY CONSUMPTION MODELLING}

In the case of a 2-level WSN, we have $\mathrm{N}_{1}$ level 1 sensor nodes (SNs) surrounding the collector node $(\mathrm{CN})$ each within the maximum efficient radio reach $\mathrm{r}$ of the $\mathrm{CN}$ and each $\mathrm{SN}$ transmitting directly to the CN. For each level 1 sensor node there is a cluster of $\mathrm{N}_{2}$ level $2 \mathrm{SNs}$ each within the maximum efficient radio reach $\mathrm{r}$ of the level 1 node and each $\mathrm{SN}$ transmitting directly to that level $1 \mathrm{SN}$. In total there are therefore $\mathrm{N}=\mathrm{N}_{1}\left(\mathrm{~N}_{2}+1\right) \mathrm{SNs}$ in this architecture. Here we will assume, as before, that the total number of $\mathrm{SNs}, \mathrm{N}$, is fixed and we have a range of options for $\mathrm{N}_{1}$.

We seek to find the best choice for the dimensionless integral quantities $\mathrm{N}_{1}$ and $\mathrm{R}$ in this architecture. Again let $\mathrm{E}_{\mathrm{S}}$ be the initial battery level when sampling operations begin at level 2 and $\mathrm{E}_{0}$ the minimal operational energy. The WSN in this architecture operates differently from the 1-level architecture of the previous section. For the 2-level architecture, the level 2 nodes sample until burnout during which the level $1 \mathrm{SNs}$ act only as relays to the $\mathrm{CN}$, and then the level 1 nodes begin sampling until burnout. A node which acts as a relay receives a signal in allocated timeslot $i$ of network cycle $j$ and stores the packet in its RAM for one cycle and then it retransmits that same packet from RAM in the same timeslot $i$ of the following network cycle $j+1$. There is no interference because SNs do not transmit in every network cycle since the SN cycle is much larger than the network cycle: $\mathrm{t}_{\mathrm{T}}>>\mathrm{T}$. It was observed in [1] that saving packets to flash memory is costly in battery life and here we are avoiding this cost by instead saving to the SN RAM. Under this scheme, the data to be relayed need only be stored in RAM for one network cycle. Since every level 1 node has $\mathrm{N}_{2}$ level 2 nodes, the nodes only need to have sufficient memory to store up to $\mathrm{N}_{2}$ data packets at a time. Receiving a transmission takes time $\Delta$ and sending a transmission takes time $\Delta$ and writing to and reading from SN RAM is assumed to take time $\Delta / \mathrm{m}$ for a large integer $\mathrm{m}$. The active and passive times for a relay during an SN cycle are therefore: 


$$
\begin{aligned}
& t_{A}^{\prime}=2(1+1 / m) \Delta \\
& t_{P}^{\prime}=t_{T}-t_{A}^{\prime}
\end{aligned}
$$

This leads tothe energy consumption per relay node per SN cycle as:

$$
E_{T}^{\prime}=\mathcal{E}_{A} t_{A}^{\prime}+\mathcal{E}_{P} t_{P}^{\prime}
$$

The level 2 nodes burn out after $\mathrm{P}_{2}=\mathrm{N}_{2}\left(\mathrm{E}_{\mathrm{S}}-\mathrm{E}_{0}\right) / \mathrm{E}_{\mathrm{T}}$ messages which takes a duration of $\mathrm{L}_{2}=\mathrm{P}_{2} \mathrm{t}_{\mathrm{T}}$.

The level 2 SNs therefore have their initial energy for sampling reduced to:

$$
E_{S}^{\prime}=E_{S}-E_{T}^{\prime} P_{2}
$$

We can now determine the WSN productivity $\mathrm{P}_{1}$ (total number of samples sent) and sampling longevity $\mathrm{L}_{1}$ (duration of the WSN after initialization) for the level 1 SNsaccording to these formulas:

$$
\begin{array}{ll}
P_{1}=N_{1} \frac{\left(E_{S}^{\prime}-E_{0}\right)}{E_{T}} & P_{2}=N_{2} \frac{\left(E_{S}-E_{0}\right)}{E_{T}} \\
L_{1}=\frac{\left(E_{S}^{\prime}-E_{0}\right) t_{T}}{E_{T}} & L_{2}=\frac{\left(E_{S}-E_{0}\right) t_{T}}{E_{T}}
\end{array}
$$

The total productivity of the WSN is $\mathrm{P}=\mathrm{P}_{1}+\mathrm{P}_{2}$ and its longevity is $\mathrm{L}=\mathrm{L}_{1}+\mathrm{L}_{2}$. A plot of productivity $\mathrm{P}$ for typical WSN parameters is shown in Figure $3 \mathrm{a}$ and the corresponding longevity $\mathrm{L}$ plot is shown in Figure $3 \mathrm{~b}$. It is clear that the productivity curve is still hyperbolic but that the longevity curve has a peak and so the WSN is now intrinsically optimizable.

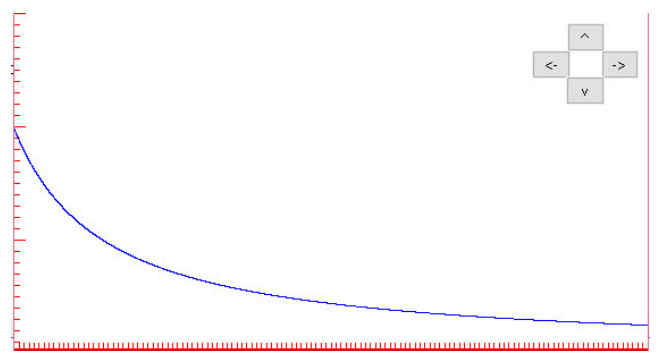

Figure 3a Productivity plot of $\mathrm{P}$ (vertical axis) against $\mathrm{R}$ (horizontal axis) in suitable units.

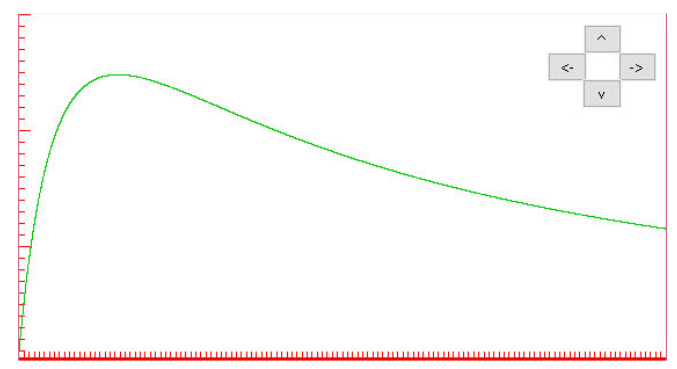

Figure $3 b$ Longevity plot of L (vertical axis) against R (horizontal axis) in suitable units. 


\section{Optimal Rest Period Versus Second LeVel Density}

To find the optimal rest period $\mathrm{R}$ as a function of the level 2 cluster size $\mathrm{N}_{2}$ we must differentiate the longevity $\mathrm{L}=\mathrm{L}_{1}+\mathrm{L}_{2}$ with respect to $\mathrm{R}$ and set $\partial \mathrm{L} / \partial \mathrm{R}$ to zero. To make the calculations more tractable we will express $L$ as a function of $t_{T}$ and $N_{2}$ rather than $R$ and $N_{2}$ and first optimize $L$ for $t_{T}=t^{*}$. Since $t_{T}$ is linear in $R$ this will also correspond to the optimization of $L$ for $R=R^{*}$ after which the optimal value in $\mathrm{N}_{2}$ is found. In terms of $t, \mathrm{~L}$ is expressed as:

$L\left(t_{T}, N_{2}\right)=\left(\frac{E_{S}-E_{0}}{\varepsilon_{P}}\right) \frac{t_{T}}{\mu_{A}+t_{T}}\left\{2-N_{2}\left(\frac{\varkappa_{A}{ }^{\prime}+t_{T}}{\mu_{A}+t_{T}}\right)\right\}$

where

$\gamma=\frac{\varepsilon_{A}-\varepsilon_{P}}{\varepsilon_{P}}$

$t_{A}{ }^{\prime}=2 N_{2} \Delta$

In this formula for $\mathrm{L}, E_{S}, E_{0}, \varepsilon_{A}, \varepsilon_{P}, \gamma, t_{A}$ and $\Delta$ are all constants and

$t_{T}=(Z+1+R) T$

where $\mathrm{Z}$ and $\mathrm{T}$ are also constants. In the partial derivative of $\mathrm{L}$ with respect to $\mathrm{t}_{\mathrm{T}}$ we are sure that the denominator $\varkappa_{A}+t_{T}$ is never infinite so we finally derive:

$t^{*}=\frac{\varkappa_{A}\left(t_{A}-t^{\prime}{ }_{A} N_{2} / 2\right)}{\left(N_{2}-1\right) t_{A}-t^{\prime}{ }_{A} N_{2} / 2}$

The second optimization is to optimize the above equation by computing $\frac{d t^{*}}{d N_{2}}$ and equating that to zero. The result is

$N_{2}^{*}=\frac{t_{A}\left(\frac{2}{t_{A}{ }^{\prime}}-1\right)}{1-t_{A}+t_{A}{ }^{\prime} / 2}$

In most cases we also have $t_{A}{ }_{A}<<t_{A}$ so that

$t^{*} \approx \frac{\varkappa_{A}}{N_{2}-1}$

The result is:

$R^{*}=\frac{\varkappa_{A}\left(t_{A}-t_{A}^{\prime} N_{2} / 2\right)}{T\left(\left(N_{2}-1\right) t_{A}-t^{\prime}{ }_{A} N_{2} / 2\right)}-Z-1 \approx \frac{\varkappa_{A} / T}{N_{2}-1}-Z-1$

$N_{2}^{*}=\frac{t_{A}{ }^{\prime}}{2 \Delta}$

As an example, for a simplified case, $\varepsilon_{A}=1.0, \varepsilon_{P}=0.01, \gamma=99, \mathrm{~T}=5 \mathrm{~ms}, \mathrm{t}_{\mathrm{A}}=0.1, \mathrm{t}_{\mathrm{A}}{ }^{\prime}=9 \times 10^{-}$ ${ }^{4}, \mathrm{t}_{\mathrm{T}}=1.21 \mathrm{~s}, \mathrm{Z}=20, \Delta=50 \mu \mathrm{s}, \mathrm{N}=100, \mathrm{~N}_{1}=10, \mathrm{~N}_{2}{ }^{*}=9$ giving $\mathrm{t}^{*}=1.24 \mathrm{~s}$ and $\mathrm{R}^{*}=229$ as the optimal rest duration of each $\mathrm{SN}$ in units of network cycle time $\mathrm{T}$. 


\section{DisCuSSION OF RESUltS}

Two programs, shown in Figures 2a and 2b, were written (in Delphi for PCs) to visually display the productivity and longevity curves for the one level and the two level networks. The results for one level WSNs (shown in Figure 2a) were as expected: the productivity decreases by increasing the system rest period $\mathrm{R}$ and the optimal performance occurs only for $\mathrm{R}=0$.Productivity decreases for increasing $\mathrm{R}$ because rest periods also consume battery energy. The longevity increases with increasing node rest duration but only to an unattainable asymptotic limit at infinite R. This leads to a trade-off: decrease $\mathrm{R}$ to increase productivity and increase $\mathrm{R}$ to increase longevity with the balance between productivity and longevity decided by the network manager rather than the architecture. The one-level WSNs thus do not give enough degrees of freedom for unique WSN performance and longevity optimization.

The results for two-level WSNs (Figures 2b, 3a and 3b) showed more interesting features as seen in the two graphs for productivity and longevity. As in the one level network, longevity is a decreasing function of $\mathrm{R}$. This reflects the intuitive consideration that the longer the time between sampling the fewer samples will be taken in the end because more battery energy is spent in the node's "passive" functions. This curve is similar to the one level network performance curve. The two level longevity curve shows a peak (highlighted by a blue line in Figure 2b) which is not present in the one level network longevity curve. The two level longevity curve shows that increasing the rest period of a node initially lengthens how long the battery will last. However this cannot be done indefinitely because passive battery consumption means that the battery cannot last indefinitely. There is therefore an optimal rest duration $\mathrm{R}^{*}$ as computed in the previous section Eqn(14) and a corresponding optimal fan out and WSN longevity $L^{*}=L\left(R^{*}\right)$ for the case of the two level networks.

\section{Conclusions}

In this work we investigated the effectiveness of hierarchical WSNs with regard to productivity and longevity. Programs were written to display the relevant productivity and longevity curves for specific WSN parameters. By concentrating on one level and then two level architectures it was found that one level architectures provided no flexibility for unique optimization determinable by the architecture and are in fact unoptimizable, but in contrast two level architectures can be optimized for longevity. The optimization involves using the best fan out $\mathrm{N}_{2}$ * from level 1 nodes and the best rest duration $\mathrm{R}^{*}$ for all SNs.

There are two directions for further research from this work. Firstly it would be good to know what optimization is possible for three and higher level hierarchical WSNs. This work suggests that such optimizations exist and that the number of parameters in the optimization is greater egfanouts $\mathrm{N}_{2}, \mathrm{~N}_{3}$ and rest duration $\mathrm{R}$ for three level WSNs.

Secondly it is apparent from the productivity curves, that all nodes should not be treated equally simultaneously. It is desirable to have a levelled off productivity curve rather than one that is continually dropping. To maintain a constant averaged productivity level for an extended operational period is therefore preferable than the design considered in this work. Such plateaued productivity requires that sampling is not done uniformly by all active SNs at the same time but rather by a smaller number of active sensors on a roughly similar spatial distribution with all available SNs sharing the sampling activity load equally on average over the longer term. These two conclusions will be considered in future research on WSN effectiveness. 
International Journal of Wireless \& Mobile Networks (IJWMN) Vol. 9, No. 3, June 2017

\section{ACKNOWLEDGEMENTS}

The author would like to thank Charles Sturt University Study Group where the research work was undertaken whilst working there.

\section{REFERENCES}

[1] Hoang AN, Forster A, Puccinelli D, Giordano S, "Sensor Node Lifetime: An Experimental Study",

[2] Yu-Chee Tseng, You-Chiun Wang, Kai-Yang Cheng "iMouse: An Integrated Mobile Surveillance and Wireless Sensor System" IEEE Computer Volume: 40, Issue: 6, June 2007.

[3] Wen-Tsuen Chen, Po-Yu Chen, Wei-Shun Lee, Chi-Fu Huang, "Design and Implementation of a Real Time Video Surveillance System with Wireless Sensor Networks", Vehicular Technology Conference, 2008. VTC Spring 2008. IEEE.

[4] Ali Benzerbadj, BouabdellahKechar,AhcéneBounceur, Bernard Pottier "Energy Efficient Approach for Surveillance Applications Based on Self Organized Wireless Sensor Networks", Procedia Computer Science, (Elzevier), Volume 63, 2015, Pages 165-170.

[5] Mahmood Ali, Annette Böhm and Magnus Jonsson, "Wireless Sensor Networks for Surveillance Applications - A Comparative Survey of MAC Protocols", In: The fourth international conference on wireless and mobile communications (ICWMC 2008). Piscataway: IEEE; 2008. p. 399-403.

[6] Muhammad Farhan Khan, Emad A. Felemban,; SaadQaisar, Salman Ali, "Performance Analysis on Packet Delivery Ratio and End-to-End Delay of Different Network Topologies in Wireless Sensor Networks (WSNs)", pp 324-329, 2013 IEEE 9th International Conference on Mobile Ad-hoc and Sensor Networks, 2013.

[7] MeenakshiTripathi, R. B. Battula, M. S. Gaur, V. Laxmi, "Energy Efficient Clustered Routing for Wireless Sensor Network”, pp 324-329, 2013 IEEE 9th International Conference on Mobile Ad-hoc and Sensor Networks, 2013.

\section{Author}

Dr J Rankin has undertaken research over 45 years starting with General Relativity and Mathematical Physics at the University of Adelaide followed by Computer Graphics, Fuzzy Logic Systems and Games Technology at La Trobe University and most recently Wireless Sensor Networks with Charles Sturt University Study Group Melbourne.

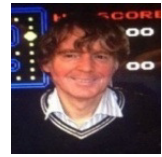

